\title{
SVM-BASED TEXTURE CLASSIFICATION IN OPTICAL COHERENCE TOMOGRAPHY
}

\author{
N. Anantrasirichai ${ }^{\star}$, Alin Achim ${ }^{\star}$, James E Morgan ${ }^{\dagger}$, Irina Erchova ${ }^{\dagger}$, Lindsay Nicholson ${ }^{\star \star}$ \\ * Visual Information Laboratory, University of Bristol, UK \\ ${ }^{\star \star}$ School of Cellular and Molecular Medicine, University of Bristol, UK \\ ${ }^{\dagger}$ School of Optometry and Vision Sciences, Cardiff University, UK
}

\begin{abstract}
This paper describes a new method for automated texture classification for glaucoma detection using high resolution retinal Optical Coherence Tomography (OCT). OCT is a non-invasive technique that produces cross-sectional imagery of ocular tissue. Here, we exploit information from OCT images, specifically the inner retinal layer thickness and speckle patterns, to detect glaucoma. The proposed method relies on support vector machines (SVM), while principal component analysis (PCA) is also employed to improve classification performance. Results show that texture features can improve classification accuracy over what is achieved using only layer thickness as existing methods currently do.
\end{abstract}

Index Terms - classification, support vector machine, optical coherence tomography, texture

\section{INTRODUCTION}

Optical Coherence Tomography (OCT) is a powerful tool in clinical practice, used to assess retinal damage in a range of diseases It provides ultrahigh-resolution cross-sectional images of biological tissues using non-invasive imaging technology. While recent OCT technology offers higher scan speed and better signal sensitivity, the spatial-frequency bandwidth of the interference signals is still limited and leads to an appearance called 'speckle'. Speckle, which appears as a grainy texture, is believed to carry information about tissue microstructure and can potentially be used for disease analysis [1].

While there are a number of works which employ texture information to classify layers of OCT images, only a few reports characterise normal and abnormal tissue of the retina through the use of texture features. For example, the method proposed in [2] determines footprints of fluid-filled regions in the macula using local texture and thickness properties across the macula. The presence of normal macula and each of three types of macular pathologies (macular edema, macular hole, and age-related macular degeneration) are identified via macula structure and textures [3]. None of these approaches have employed texture for glaucoma detection.

This work was partly supported by the EPSRC through the Bio-E Initiative of the Severnside Alliance for Translational Research (SARTRE).
The assessment of glaucoma disease using OCT image, so far, has focused on the delineation of retinal layers or emphasised the detection of cell loss and anatomical disruption in the retinal architecture. OCT images can reveal thinning of the retinal nerve fibre layer (RNFL) and retinal ganglion cells (RGCs) [4]. In [5], the RNFL thickness profile of a single circular OCT B-scan is employed for automated glaucoma classification. Recently, evidence has been presented that many retinal conditions first develop as a loss of neuronal connectivity and damage to the RGC/IPL complex (the combined RGC and inner plexiform layers (IPL)) [6]. The work in [7] shows that the light scattering properties of retinal layers, affected by retinal neural atrophy, can be detected by OCT. Therefore, we hypothesise that texture due to light reflections within given retinal layers can be used for detecting glaucoma.

In this paper, we propose an automated glaucoma classification method using OCT images. Not only is the thickness of the retinal layers employed as do existing approaches, but also the texture information of those layers is extracted and utilised as features in a classifier. The method is developed using support vector machines (SVM) [8]. This is a supervised learning algorithm that analyses data by constructing a set of hyperplanes used for separating different classes of data in the feature space. The problem can be simply modelled as a linear classifier; however, it may happen that the sets to discriminate are not linearly separable in that space. In this case, SVM provides a straightforward implementation using a nonlinear kernel function, e.g. polynomial, radial basis function (RBF), sigmoid, etc. More details on the usage of SVM can be found in [8].

The remaining part of this paper is organised as follows. The proposed scheme for automated glaucoma classification is described in detail in Section 2. The performance of the method is evaluated and discussed in Section 3. Finally, Section 4 presents the conclusions of the study.

\section{PROPOSED APPROACH}

The block diagram of the proposed SVM-based texture classification in OCT is illustrated in Fig. 2. Two major groups of features are extracted, i.e. retinal layer thickness and IPL 

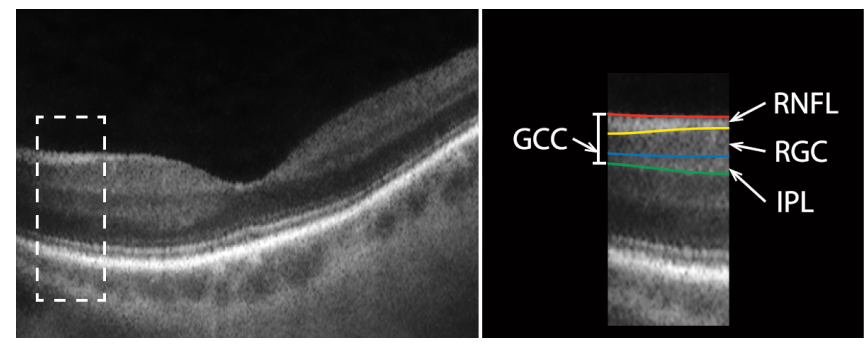

Fig. 1. OCT image showing the macula. Left: B-scan ( $x z$ plane) showing fovea. Right: Innermost retinal layers.

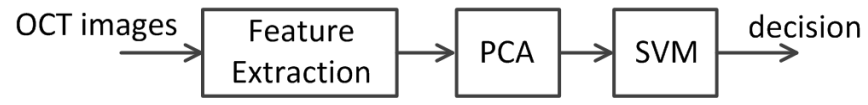

Fig. 2. Proposed SVM-based texture classification.

texture from OCT images. Before applying SVM, we always perform principal component analysis (PCA). This is known to improve classification performance. The various classes of features that were employed in this study are briefly outlined in the following.

\subsection{Retinal Layer Thickness}

OCT images reveal the inner retinal layers, which are particularly affected in glaucoma. We generate 3 features using the average thickness of the 3 innermost retinal layers, namely RNFL, IPL and ganglion cell complex (GCC) comprising the RNFL, RGC and IPL. These layers are shown in Fig. 1. Note that we employ the thickness of GCC, instead of RGC, since the thickness of the RGC in some areas is very small and difficult to measure correctly. We note here that there are a number of automatic layer segmentation methods proposed for OCT images which can be employed for extracting the layer thickness. However, to avoid errors in layer thickness measurement, we manually checked the results of the segmentation technique we used (proposed in [9]). Layer segmentation is therefore not discussed in this paper.

\subsection{Texture Features}

Speckle is the effect generated by the interference of waves with random phases. The reflected beams form some dark and bright spots with varying patterns. This can be interpreted as 'texture' on the image. Texture is an efficient tool to characterise various properties, such as structure, orientation, roughness, smoothness, or regularity differences of diverse regions in an image. We extract the textural features from IPL of OCT images. The features used in our work are tabulated in Table 1 and their details are described below. It should be noted that all texture feature extraction is performed on 2D slices. The main reason is that the 3D OCT data is captured as a series of slices corresponding to a sequence of $x z$ scans. During the acquisition of the entire image, misalignment across slices
Table 1. List of features used in classification study

\begin{tabular}{lc}
\hline Features & \# dimension \\
\hline Intensity level distribution (ILD) & \\
Mean, Variance, Skewness, Kurtosis, Entropy & 5 \\
Run-length measures (RLM) & \\
Short/Long run emphasis & 2 \\
Grey-level/Run-length non-uniformity & 2 \\
Run Percentage & 1 \\
4 directions of above features & 20 \\
Grey-level co-occurrence matrix (GLCM) & \\
Energy, Correlation, Inertia, Entropy & 4 \\
Cluster Shade, Homogeneity & 2 \\
Inverse difference moment & 1 \\
4 directions of above features & 28 \\
Complex wavelet transform (CWT) (2 levels) & \\
Mean, Variance of magnitudes & 4 \\
Mean, Variance of phases & 4 \\
Mean of magnitudes of each sub-band & 12 \\
Co-occurrence matrix of lowpass & 14 \\
Run-length measures of lowpass & 10 \\
Local Binary Pattern (LBP) & \\
Histogram & 59 \\
Granulometry (GRA) & \\
Histogram & 12 \\
\hline
\end{tabular}

can occur due to the natural and involuntary movement of the subject's eyes. This is shown as the rough surface in the $x y$ planes and zigzag lines in the $y z$ planes in Fig. 3 right.

\subsubsection{Intensity level distribution}

Five parameters are extracted from the intensity level distribution: mean measuring average of intensity values; variance measuring contrast of grey level intensities; skewness measuring asymmetry of the distribution; kurtosis measuring peakedness of the distribution and entropy measuring randomness.

\subsubsection{Run-length measures}

Run-length measurement (RLM) calculates the number of occurrences of a run with a specific length and specific grey level in a given direction. Texture properties obtained from RLM include: long run emphasis measuring coarse texture; short run emphasis measuring fine texture; run length nonuniformity measuring similarity of the run lengths; grey-level non-uniformity measuring similarity of grey level values; run percentage measuring the number of short runs. These features display the tonal distribution and heterogeneity of the OCT image. Four directions comprising horizontal, vertical and two diagonal directions are used to compute RLM. 20 features are obtained from each direction and 5 features come from their average values, since texture in the IPL of glaucoma eyes may or may not be affected directionally.

\subsubsection{Grey-level Co-occurrence matrix}

Grey-level co-occurrence matrix (GLCM) describes the spatial relationship between each intensity tone by considering 
changes between grey levels $i$ and $j$ at a particular displacement distance $d$ and at a particular angle $\theta$. Seven properties from GLCM are computed, i.e. energy measuring uniformity of local grey scale distribution; correlation measuring the joint probability of occurrence; inertia or contrast measuring the local variations; entropy measuring randomness; cluster shade measuring a group of pixels that have similar grey level values; homogeneity measuring the closeness of the distribution and inverse difference moment measuring local minimal changes. Here, we use $d=1$ and $\theta=$ $\left\{0^{\circ}, 45^{\circ}, 90^{\circ}, 135^{\circ}\right\}$ to extract 28 features, and also utilise the average values of all angles to obtain 7 features.

\subsubsection{Wavelet features}

One of the most important aspects of texture is scale, so a multiresolution approach is utilised. Here we employ the Dual Tree Complex Wavelet Transform (DT-CWT) [10] to extract texture features in different scales. Not only does the transformed image contain both spatial and frequency information, but beyond the traditional discrete wavelet transform (DWT), it also provides near shift-invariance and good directional selectivity. The magnitude of highpass coefficients relates to details, while the phase of the complex wavelet coefficients corresponds to the precise location of directional features. We therefore use both information by computing their mean and variance. When two decomposition levels are used, a total of 8 features are extracted (4 from magnitudes and 4 from phases). Again, we do not yet know that the change in the texture caused by glaucoma happens differently in different orientations. Hence, we also generate the mean of the magnitudes of highpass coefficients from each individual sub-band, giving 12 features for 2 decomposition levels. Each iteration of the decomposition, the properties of RLM and GLCM of the lowpass sub-band are calculated in the similar way to 2.2.2 and 2.2.3, respectively.

\subsubsection{Local binary pattern}

Local binary pattern (LBP) labels the pixels of an image by thresholding the neighbourhood of each pixel and considers the result as a binary number [11]. Uniform patterns, a simple rotation-invariant descriptor, are generated using 8 sampling points on a circle of radius 1 pixel. There are a total of 256 patterns, 58 of which are uniform, which yields 59 different labels. A histogram with 59 bins is obtained. Frequency of each bin is used as one feature, leading to a total of 59 features.

\subsubsection{Granulometry}

Granulometry measures particle size distribution in a collection of grains by using morphological opening operation with various sizes. A histogram is then generated according to the number of isolated areas left over after applying opening operation. Here, we use the first 12 bins as texture features, since they are the majority of particle sizes.

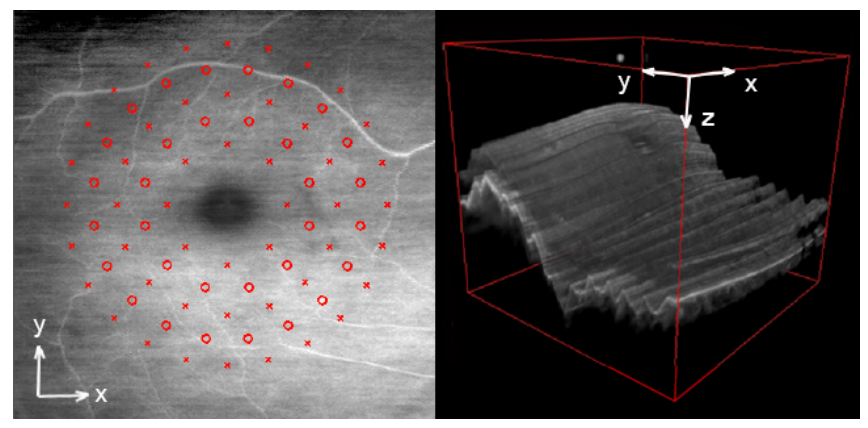

Fig. 3. Left: OCT photography shows the centres of 80 volumes used for feature extraction (The middle of the image seen as the dark spot is fovea). Right: 3D OCT volume without stack registration.

\subsection{Principal component analysis}

Principal component analysis (PCA) is a procedure which uses an orthogonal transformation to convert a set of features into a set of values of linearly uncorrelated variables. It has been used with classification problems in order to condense rich information, e.g. in SVM, PCA is sometimes used for dimensionality reduction. Redundant information is eliminated by transforming the original dataset in terms of eigenvectors. A few top eigenvalues of the covariance matrix of these data indicate the most separated vectors in the transformed space and are used as feature vectors in SVM process. Although dimensionality reduction can cause some loss of information, the transformed data can be more compact and reasonably leading to improvement of classification performance. Moreover, the computational burden of SVM classifier is significantly reduced.

\section{RESULTS AND DISCUSSIONS}

We tested our classifier on 24 retinal OCT images acquired from 14 normal eyes and 10 eyes with glaucoma. The OCT images used in this paper are 3D stacks of size $512 \times 512 \times 1024$ pixels. They are displayed on a grey scale with 16 bits/pixel. We randomly selected 7 normal eyes and 5 glaucoma eyes for training and used the rest for testing. Experiments with different random training and testing dataset were repeated 100 times and the quantitative results shown in this section constitute average results. As the visual field tests were not available, the areas of dysfunction in the eyes are unknown. We therefore exploited the global parameters by averaging the values of features extracted from 80 subvolumes from each OCT image. The size of each subvolume is $30 \times 30 \times T_{k}$ pixels, where $T_{k}$ is the thickness of IPL of subvolume $k \in\{1,2, \ldots, 80\}$. The positions of these subvolumes are shown in Fig. 3, which is the 'OCT photography' corresponding to a top view of the 3D OCT image.

We employed LIBSVM for MATLAB platform [8]. Two basic kernels were tested, linear and RBF. The parameters used in the RBF are selected by grid search using cross valida- 
Table 2. Classification accuracy using different combinations of features

\begin{tabular}{lcc}
\hline \multirow{2}{*}{ Features } & \multicolumn{2}{c}{ Accuracy \% } \\
\cline { 2 - 3 } & Linear SVM & RBF SVM \\
\hline Layer thickness only & 72.28 & \\
RNFL & 64.70 & 66.07 \\
IPL & 77.21 & 79.65 \\
GCC & 72.58 & 74.12 \\
RNFL+IPL & 80.23 & 81.07 \\
RNFL+GCC & 81.95 & 80.52 \\
All layers & & \\
\hline Texture features only & 71.65 & 72.52 \\
ILD & 62.89 & 53.32 \\
RLM & 62.48 & 58.93 \\
GLCM & 63.18 & 57.72 \\
CWT & 69.92 & 63.51 \\
LBP & 63.28 & 53.13 \\
GRA & 70.92 & 65.25 \\
ILD+LBP & 65.81 & 58.88 \\
CWT+LBP & 65.20 & 58.71 \\
ILD+CWT+LBP & 62.83 & 58.74 \\
All textures & & \\
\hline Layer thickness \& Texture & 82.98 & 82.62 \\
layers+ILD & 81.16 & 82.60 \\
layers+RLM & 82.39 & 82.08 \\
layers+GLCM & 81.48 & 83.31 \\
layers+CWT & 84.06 & 84.13 \\
layers+LBP & 81.39 & 81.84 \\
layers+GRA & 82.48 & 84.19 \\
layers+CWT+LBP & 81.28 & 84.44 \\
layers+RLM+CWT+LBP & 81.36 & $\mathbf{8 4 . 8 8}$ \\
layers+GLCM+CWT+LBP+GRA & 80.94 & $\mathbf{8 5 . 1 5}$ \\
layers+RLM+GLCM+CWT+LBP+GRA & 80.91 & 84.69 \\
layers+All texture & & \\
\hline & &
\end{tabular}

tion. The classification performance in Table 2 clearly shows that the linear SVM significantly outperforms the RBF SVM. The influence of PCA is tested and we found that applying PCA to low-dimensional feature set deteriorates the classification accuracy as it significantly misses on valuable information. Therefore, the results of using only retinal layer features shown in the first part of Table 2 were obtained by not employed PCA. In contrast, when texture features are employed, experimental results reveal the benefits of using PCA in combination with SVM, so the second and third parts of Table 2 show the results obtained by employing PCA. Although SVM seems to be inherently capable of handling high-dimensional data, PCA can improve performance by removing correlations between variables and possibly removing outliers.

Although numerous studies on glaucoma detection employ inner retinal layers, none of them employs the combination of these layers in a classification problem. Results reveal that using thickness of all three innermost layers gives the best classification accuracy $(81.95 \%)$. Using texture features gives worse results, with approximately $70 \%$ accuracy. However, combining the texture features with the layer thickness improves the classification performance to $85.15 \%$. Interest- ingly, LBP features show the most benefit to the classification performance, which is even better when other features are included.

\section{CONCLUSIONS}

This paper presents an automated texture classification for glaucoma detection using SVM-based learning method. The thickness of inner retinal layers and texture are employed. Results show that the texture features can improve the classification accuracy over using only layer thickness alone by up to $4 \%$. This may also confirm that speckle, which we interpret as texture, carries useful information about the changes caused by glaucoma in retinal tissue. For future work, texture in other retinal layers will be studied, and the information from visual fields will be used for early glaucoma detection.

\section{REFERENCES}

[1] M. Bashkansky and J. Reintjes, "Statistics and reduction of speckle in optical coherence tomography," Optics Letters, vol. 25, no. 8, pp. 545-547, 2000

[2] G. Quellec, Kyungmoo Lee, M. Dolejsi, M.K. Garvin, M.D. Abramoff, and M. Sonka, "Three-dimensional analysis of retinal layer texture: Identification of fluid-filled regions in SD-OCT of the macula," Medical Imaging, IEEE Trans on, vol. 29, no. 6, pp. 1321 -1330, 2010.

[3] Y.Y. Liu, M. Chen, H. Ishikawa, G. Wollstein, J.S. Schuman, and J.M. Rehg, "Automated macular pathology diagnosis in retinal OCT images using multi-scale spatial pyramid and local binary patterns in texture and shape encoding," Medical Image Analysis, vol. 15, no. 5, pp. 748 $-759,2011$.

[4] M. Hangai A. Sakamoto and, M. Nukada, H. Nakanishi, S. Mori, Y. Kotera, R. Inoue, and N. Yoshimura, "Three-dimensional imaging of the macular retinal nerve fiber layer in glaucoma with spectral-domain optical coherence tomography," Invest. Ophthalmol. Vis. Sci., vol. 51, no. 10 , pp. 5062-5070, 2010.

[5] M.A. Mayer, J. Hornegger, C.Y. Mardin, F.E. Kruse, and R.P. Tornow, "Automated glaucoma classification using nerve fiber layer segmentations on circular spectral domain oct b-scans," in The Association for Research in Vision and Ophthalmology, Inc., 2009.

[6] MJ Gastinger, AR Kunselman, EE Conboy, SK Bronson, and AJ. Barber, "Dendrite remodeling and other abnormalities in the retinal ganglion cells of ins2 akita diabetic mice," Invest Ophthalmol Vis Sci., vol. 49, no. 6, pp. 2635-2642, June 2008.

[7] G. Wollstein, L.A. Paunescu, T.H. Ko, J.G. Fujimoto, A. Kowalevicz, I. Hartl, S. Beaton, H. Ishikawa, C. Mattox, O. Singh, J. Duker, W. Drexler, and J. S. Schuman, "Ultrahigh-resolution optical coherence tomography in glaucoma," Ophthalmology, vol. 112, no. 2, pp. 229 237, 2005.

[8] C.C. Chang and C.J. Lin, "LIBSVM: A library for support vector machines," ACM Transactions on Intelligent Systems and Technology, vol. 2, pp. 27:1-27:27, 2011.

[9] R.J. O'Callaghan and D.R. Bull, "Combined morphological-spectral unsupervised image segmentation," Image Processing, IEEE Transactions on, vol. 14, no. 1, pp. 49-62, Jan. 2005.

[10] I.W. Selesnick, R.G. Baraniuk, and N.G. Kingsbury, "The dual-tree complex wavelet transform," Signal Processing Magazine, IEEE, vol. 22, no. 6, pp. 123 - 151, Nov. 2005.

[11] T. Ojala, M. Pietikainen, and T. Maenpaa, "Multiresolution gray-scale and rotation invariant texture classification with local binary patterns," Pattern Analysis and Machine Intelligence, IEEE Transactions on, vol. 24, no. 7, pp. $971-987$, Jul. 2002. 